\title{
IL CARME DEI SEPOLCRI DI UGO FOSCOLO IN DIALOGO CON L'ELEGIA À MEMÓRIA DO PRESIDENTE-REI SIDÓNIO PAIS DI FERNANDO PESSOA: IL TRIONFO DELLA POESIA SULLA MORTE
} Valentina Bosi ${ }^{\star}$

Riassunto: Il presente lavoro propone l'analisi comparata del carme Dei Sepolcri scritto dal poeta italiano Ugo Foscolo e dell'opera Alla memoria del Presidente-Re Sidónio Pais, una delle più importanti elegie mai scritte in portoghese, che Fernando Pessoa ha consegnato al linguaggio della poesia. Entrambi gli autori si muovono dagli avvenimenti della contemporaneità per poi riflettere più generalmente sul significato della morte, i cui limiti possono essere valicati dalla memoria delle gesta eroiche e dalla poesia che le può rendere eterne, nella speranza di una rinascita del paese e dell'umanità.

Parole chiave: Ugo Foscolo. Fernando Pessoa. Letteratura portoghese.

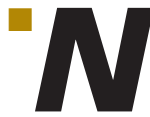
ella poesia di Fernando Pessoa (1888-1935) si incrociano non solo diverse culture, ma anche epoche molto distanti: l'innovazione si sposa con la tradizione e il passato, e proprio per questo non risulta strano avvicinare la sua opera a quella dello scrittore italiano Ugo Foscolo, grande innovatore della lirica italiana dell'Ottocento. La possibilità di stabilire un dialogo, in questo caso tra letteratura portoghese e italiana, presuppone di certo un punto di incontro, ma anche la necessaria coscienza delle differenze come grande arricchimento e come stimolo per una riscoperta della poesia nella nostra epoca post moderna.

Una complessa trama di richiami intertestuali è presente, nello specifico, all'interno della celebre elegia Alla memoria del Presidente-Re Sidónio Pais, nella quale si riflette sulla situazione del Portogallo che ha perduto ormai la sua gloria. Questo tema si allarga poi fino ad includere una più ampia riflessione sul significato della morte, la quale sembrerebbe escludere in un primo momento 
ogni possibilità di sopravvivenza di ciò che è umano e terreno. L'elegia si inserisce perciò in una più generale teorizzazione della decadenza attuale del Portogallo dopo la morte di Cardoso de Silva Sidónio Pais, il quale aveva instaurato tra il giorno 8 dicembre 1917 ed il 14 dicembre 1918 (giorno del suo assassinio) un regime di tipo autoritario e presidenzialistico, ovvero una dittatura non repressiva o totalitaria, incarnando in questo modo la guida a cui auspicavano due miti prettamente portoghesi: quello del V Impero e quello del Sebastianismo messianico. Dopo la morte di Sidónio Pais, dunque, anche Pessoa auspica la venuta del Desiderato, che avrebbe coperto il trono del Portogallo ponendosi al comando di tutta la cristianità. Nell'attesa della rinascita del suo paese, il poeta crea una sorprendente composizione poetica dove Pais viene presentato come eroe a incarnare la volontà divina. L'eletto può sopravvivere alla morte stessa, nella memoria delle sue doti e della lealtà manifestate durante la vita terrena. Segue una lunga e profonda riflessione sulla possibile sopravvivenza dell'essere umano nell'amore e nella memoria dei vivi, legata evidentemente ad una tesi sulla base di tutta la tradizione letteraria classica, che rivendica l'importanza delle azioni di ogni essere umano in grado, in questo modo, di guadagnarsi l'immortalità.

Anche il carme Dei Sepolcri di Ugo Foscolo (1778-1827), composto da 295 versi, vuol dare una risposta chiara alla domanda sul senso della morte, questione secolare da sempre dibattuta. Questi versi innalzano definitivamente Ugo Foscolo accanto ai sommi poeti, e rappresentano la prima voce lirica di una nuova letteratura (DE SANCTIS, 2002, p. 946). Prima di entrare nel dettaglio della composizione lirica, è interessante soffermarsi per brevi cenni sulla vicenda biografica del poeta Ugo Foscolo, assai differente rispetto a quella degli scrittori italiani contemporanei, a partire dalla sua nascita da madre greca nell'isola di Zacinto: la sua frequente celebrazione della classicità si colora, perciò, di tinte autobiografiche. Dopo la morte del padre, Ugo e la madre si traferiscono a Venezia, che diventa la sua seconda patria negli anni 1793-1797. Qui Foscolo compone la celebre Ode a Bonaparte liberatore: dopo il trattato di Campoformio, con il quale Bonaparte cede Venezia all'Austria, il poeta vive una forte delusione e abbandona la sua seconda patria trasferendosi a Milano. La vita di Ugo Foscolo è in numerose occasioni caratterizzata dal trauma dell'esilio, che assume tinte profondamente esistenziali. Dopo varie peregrinazioni il poeta si stabilisce a Londra fino all'anno della sua morte, luogo in cui vivevano numerosi patrioti ed esuli in forte polemica con l'Italia contemporanea. Dopo l'Unità d'Italia, le spoglie di Ugo Foscolo sono state collocate nella chiesa di Santa Croce, da lui celebrata nel carme che prenderemo in esame nel dettaglio.

I 295 versi di tale composizione lirica si dispongono secondo una vera e propria struttura argomentativa che, partendo dalla negazione di una tesi, vuole offrire una soluzione chiara al mistero della morte. Dare una risposta a tale quesito non equivale ad eliminare ogni ombra legata all'angoscia della morte, nel carme foscoliano come nell'elegia pessoana, composta in totale da 240 versi, nei quali si celebra l'importanza della memoria. Anche l'incipit del testo portoghese non è esente dal dubbio e dal dolore: l'immagine della morte che ci viene disegnata nelle prime strofe da Pessoa sembra rappresentare un totale oblio che nulla lascia dietro di sé.

Gli episodi della contemporaneità di entrambi i poeti fungono da stimolo iniziale per la creazione dei poemi, che nonostante questo non possono generica- 
mente essere considerati come poesia di occasione, coinvolgendo una riflessione in realtà molto più ampia e profonda. Accanto alla morte di Sidónio Pais a cui fa riferimento Pessoa si pone infatti la reazione del poeta italiano Foscolo all'ingiustizia dell'editto di Saint-Cloud (del 1804) con il quale veniva disposto che le tombe fossero collocate fuori dalle città e avessero lapidi uguali. Questa è la sollecitazione esterna alla composizione del carme, nel quale si riflette poi profondamente a livello esistenziale.

Molto interessante risulta seguire la logica consequenziale secondo la quale si articolano le due opere, secondo un vero e proprio schema di trionfi sulla morte nel carme foscoliano. In sintesi, gli affetti e la memoria civile vincono l'oblio dell'essere umano, quando l'elemento di continuità tra i vivi e i morti è stabilito dalla tomba. Purtroppo, il tempo potrebbe minacciare la conservazione del sepolcro: l'unica forza ritenuta da Foscolo realmente in grado di eternare ciò che è semplicemente umano e finito è quella della poesia.

In particolare, il carme Dei Sepolcri si apre con una riflessione dolorosa sull'impossibilità di evitare l'oblio del defunto, partendo dalla negazione della tesi principale, come in una dimostrazione matematica: più affascinante sarà il rinascere dei fiori dopo un duro inverno, portando con sé una nuova primavera. Si afferma di seguito, infatti, che quando si è privati dell'incanto della natura, della speranza del futuro e dei propri affetti, il sepolcro non può modificare il destino del defunto di oblio e annullamento. Anche Pessoa (2010, p. 34, v. 4), nei primi versi dell'elegia, descrive ogni fama e valorosa battaglia ormai lontane dal defunto: "só a noite enorme", afferma il poeta, domina in tale universo. Le ombre angoscianti della morte sono diffusamente presenti anche nel testo italiano, ove si afferma che nulla può porre fine a "l'alterna onnipotenza delle umane sorti" (FOSCOLO, 2001, p. 336, v. 182-183). Inoltre, a suggello del carme, si pone un verso che accomuna l'impegno, la lotta e i sentimenti dell'uomo nella pessimistica definizione di "sciagure umane" (FOSCOLO, 2001, p. 341, v. 295). Tali connotazioni contrastanti della morte diffondono una certa ambivalenza nel poema, poiché quello che si afferma sul piano razionale non sembra sempre del tutto acquisito sul piano emotivo: la paura della fine tradisce talvolta la generale affermazione della sopravvivenza nel ricordo. Anche nell'elegia, proseguendo nella lettura, si introducono dubbi e incertezze, fino ad annunciare una triplice vittoria sulla morte: da un punto di vista esistenziale, poiché ad esaurirsi è solamente il corpo, in senso storico, potendo il Portogallo ancora rinascere, e in senso occulto, riferito al mito perpetuante del Sebastianismo. Questo avviene poiché "a alma acesa não aceita essa morte absoluta” (PESSOA, 2010, p. 36, v. 29-30), inaugurando per mezzo di una rivolta dello spirito una riflessione più ampia.

Dopo un totale pessimismo riguardo la condizione umana si giunge, tanto nel testo italiano quanto in quello portoghese, ad un primo trionfo sulla morte: la vittoria degli affetti sull'oblio, sintetizzata da Pessoa (2010, p. 39) nell'espressione "falsa morte". L'immagine foscoliana delle Ore che danzano intorno al sole (FOSCOLO, 2001, p. 327, v. 6-7) rappresenta l'inesorabile trascorrere del tempo, a causa del quale non si deve però rinunciare alla speranza della sopravvivenza: la continuità degli affetti, il colloquio con i vivi può salvare i defunti dall'oblio e dall'annullamento. All'annientamento fisico si contrappone infatti l'intensità del ricordo nei vivi, assicurando ai morti una prima forma di immortalità. In questo senso, afferma infatti Pessoa (2010, p. 36, v. 35-36): "Quão mais crê o Rei ainda existente / O amor de um povo!", permettendo al sovrano quasi il superamento 
dei limiti della condizione umana. Il poeta afferma di seguito: "A Vida fel-o heroe, e a Morte / o sagrou Rei!" (PESSOA, 2010, p. 36, v. 39-40), stabilendo così una continuità profonda e logica tra vita e morte, che non è altro che la sua prosecuzione in altra forma. Il defunto è infatti "No occulto para o nosso olhar / no visivel à nossa alma” (PESSOA, 2010, p. 36, v. 45-46), poiché la corrispondenza degli affetti oltre la morte garantisce l'unica sopravvivenza che conti. Anche il Foscolo, all'interno della sua argomentazione, dichiara: "[...] Celeste è questa / corrispondenza d'amorosi sensi / celeste dote è negli umani; e spesso / per lei si vive con l'amico estinto / e l'estinto con noi" (FOSCOLO, 2001, p. 328-329, v. 29-33). Si tratta, nel testo italiano, di un'immagine molto suggestiva descritta all'ombra di un albero amico, che con il profumo dei suoi fiori e l'ombra rinfrescante che sparge consola le spoglie del defunto. In questi versi, l'uso del latinismo "Arbore" (FOSCOLO, 2001, p. 329, v. 39) conferisce sensibilità femminile e umana alla natura, la quale accoglie l'uomo porgendogli l'estremo rifugio. In molti passi del carme a seguire il verde sarà il colore predominante: al mondo classico appartiene l'immagine dei cipressi e degli alberi che con le loro fresche fronde rendono perenne la memoria dei defunti. Lo stesso avviene nella descrizione dei giardini dei cimiteri inglesi, che allo stesso modo rendono eterna la memoria secondo la duplice prospettiva pubblica e privata. Anche il carme del Foscolo inventa una nuova immortalità, una storia dell'umanità proposta da un punto di vista diverso: una storia dei vivi costruita dai morti (DE SANCTIS, 2002, p. 947). I principi della religiosità tradizionale interessano ben poco, infatti, il Foscolo (come la dannazione delle anime o la beatitudine sotto le grandi ali del perdono di Dio): in questo caso quello che interessa è una sopravvivenza tutta laica, la realizzazione o meno della corrispondenza di amorosi sensi.

La tomba non è, però, di consolazione per tutti, ma solo per chi ha saputo conquistarsi l'amore degli altri e l'eternità nel loro ricordo: si introduce, in questo senso, una concezione assai elitaria della sopravvivenza, all'interno dei testi portoghese ed italiano. In questo senso la figura dell'eroe, eternamente presente nella memoria, fa da protagonista nelle due opere prese in esame, quando Pessoa (2010, p. 38, v. 67-68) afferma "Não passa como o vento o heroe / sob o ermo céu", riscattando l'eroe dalla sua esistenza solamente terrena. E di seguito anche nel carme foscoliano si enuncia l'importanza del "nome" dell'eroe, da rendere evidente proprio nel luogo del sepolcro: al contrario l'editto di Saint-Cloud vorrebbe eguagliare le tombe rendendole inumane, da cui scatta la furiosa denuncia del Foscolo. Così la città di Milano, dominata ormai da vuoti interessi mondani, dimentica i suoi eroi nelle cosiddette "obliate sepolture" (FOSCOLO, 2001, p. 330, v. 51-53), senza dedicare una lapide al grande scrittore Giuseppe Parini. La privazione del nome provoca una profonda sofferenza in Ugo Foscolo, poiché come insegna il Logos greco nominare le cose equivale a conoscerle. Il nome e la fama sono dunque portatori di identità, e la sopravvivenza meritocratica di cui parla il poeta italiano richiama la figura dell'eroe che troviamo in Pessoa. Di fronte a tale oblio, il poeta portoghese smarrisce per un attimo la speranza, perdendo anche la sua fede, in un vero e proprio desconforto do mundo in senso esistenziale e storico, riferito alla condizione del suo paese. La speranza può rinascere solo nella memoria dei grandi e delle loro gesta, da rendere ben evidenti nei luoghi della sepoltura secondo Foscolo (2001, p. 331, vv. 70), che il decreto di Saint-Cloud rendeva "plebei tumuli" della folla anonima. Si descrive così nel carme, con tratti volutamente lugubri, lo squallido cimitero in cui 
giace il Parini, sorvolato dall'upupa, ritenuta un pauroso uccello notturno per un errore risalente al commento della Bibbia, nonché al mito narrato da Ovidio secondo il quale l'upupa si ciberebbe di carne umana. Il poeta Eugenio Montale riscatterà, nel secolo XX, l'immagine dell'upupa, "uccello calunniato dai poeti" (MONTALE, 2001, p. 61, v. 82). Tale cimitero lugubre descritto da Foscolo diviene metafora del vuoto di speranza: non possono nascere fiori su delle tombe ignote, ove non vi sia quella "corrispondenza d'amorosi sensi" (FOSCOLO, 2001, p. 328, v. 29-33) ampiamente presente anche nel testo portoghese come unico riscatto tra le macerie di una nazione in attesa della rinascita. I Sepolcri divengono luoghi deputati alla celebrazione del passato da cui trarre stimoli per il presente, nella loro doppia funzione privata e civile, rivelando una concezione elitaria della vita e della morte: questa è anche la funzione delle tombe nella chiesa di Santa Croce a Firenze, che diviene vero e proprio mito nel testo italiano. La miseria del presente suscita anche in Pessoa un vago sentimento misto tra nostalgia e speranza per il futuro, assimilata al mito tutto portoghese del V Impero. Si materializza così il sogno di un nuovo sovrano, "o Desejado" (PESSOA, 2010, p. 52, v. 239), che possa risvegliare nel cielo una nuova aurora. In questo senso, $\mathrm{i}$ versi portoghesi contengono un ulteriore elemento rispetto al carme Dei Sepolcri, disegnando un vero e proprio progetto di rinascita per il Portogallo dopo la morte di Sidónio Pais: si ha la premonizione di un'alba non molto lontana.

La memoria delle vicende a cui presero parte i grandi eroi pervade i luoghi che ne sono stati teatro, come Al-Ksar el Kebir nella notte della celeberrima battaglia (PESSOA, 2010, p. 48, v. 169-170) che vide la grave disfatta dell'esercito portoghese contro quello marocchino. Tra i soldati venne ucciso anche D. Sebastião, il cui corpo non fu mai ritrovato, alimentando la leggenda sul futuro ritorno del sovrano, sinonimo dell'attesa di rinascita e ripresa. Anche nel carme foscoliano la memoria delle grandi gesta perdura nei luoghi che ne sono stati teatro: come nei campi di Maratona (FOSCOLO, 2001, p. 337, v. 199-201) ove si svolse la celebre battaglia che vide i greci contro i persiani (490 a.C.). Il Foscolo afferma che chiunque si ritrovi a passare per quei luoghi può ancora udire il nitrire dei cavalli, il pianto dei moribondi, o intravedere i fantasmi dei combattenti. Si viene così a delineare uno scenario molto suggestivo che scardina le leggi naturali per far convivere il passato con il presente, ove l'universo assorbe ed immortala ciò che deve essere ricordato.

La memoria dei grandi investe dunque i luoghi e i sepolcri, che possono però subire l'azione del tempo: è soprattutto la poesia in verità che vince l'azione distruttiva del tempo, trionfando sui secoli ed eternando ciò che deve permanere nella memoria. In particolare, nel testo portoghese i "clarins" (PESSOA, 2010, p. 52, v. 227) risuonano acclamando il Desiderato, evidente metafora del risuonare della poesia in un'epoca di ottenebramento. Attraverso la celebrazione della poesia, entrambi gli autori concludono i componimenti con una nota di speranza e rinascita. Foscolo completa il suo schema di trionfi sulla morte designando la funzione eternatrice della poesia: lo scrittore è solo un tramite di questo disegno, invocando nel finale le Muse per cantare le gesta dei grandi uomini. E le Muse vengono descritte come le custodi dei Sepolcri: quando il tempo, con la sua inesorabile azione, ne cancellerà persino le macerie, le Dee allieteranno con il loro canto quei luoghi, intonando una musica al di sopra del tempo che condanna ogni cosa al silenzio. 
Molto interessante è che anche nelle figure mitiche, come nel caso di Omero, Foscolo instilla sentimenti profondamente umani, allargando ancora di più questo rapporto vivi-morti nella corrispondenza di amorosi sensi che lui, in quanto poeta, può rendere eterna.

Dal punto di vista stilistico e metrico, entrambi i componimenti presi in esame sfoggiano una lingua ed un tono elevati, rispettando il principio aristotelico secondo il quale la forma deve adattarsi al contenuto: ad una materia alta perciò corrisponde una forma elevata. Così allo stile altisonante del Foscolo, che riflette sulla possibilità di una sopravvivenza oltre la morte, corrisponde il tono elevato dell'elegia di Pessoa, permeata dalla speranza di una rinascita della nazione, di cui il poeta può predire il futuro politico. Il grande ruolo del "poeta-vate" viene ricoperto da Fernando Pessoa e Ugo Foscolo, impegnati in una riflessione che trascende i limiti umani. La parola poetica è in questo modo talmente carica di contenuto da bastare a se stessa: si annuncia così una nuova letteratura come soppressione dello schema rigido della rima e come utilizzo del verso sciolto. E nel caso di Ugo Foscolo in particolare, anche il genere appare nuovo, poiché al sonetto e alla canzone si sostituisce il carme, forma libera da ogni esterno meccanismo, nell'utilizzo dell'endecasillabo sciolto che prevale poi in tutta la poesia del secolo XX. Occorre infatti la massima libertà per poter cantare la vita intera di ogni uomo, nella sua esistenza civile di patriota e cittadino e nell'intimità dei suoi affetti familiari. L'uso frequente dell'enjambement, in Foscolo e in Pessoa, permette flessibilità del verso nella possibilità di creare sequenze, allungare o accorciare il periodo, valorizzare una parola anziché un'altra. Collegabile all'enjambement è un ricorso frequente alla tecnica dell'inversione, mediante la quale la nuova collocazione della parola va a scardinare l'ordine logico del discorso, definendo delle espressioni assai connotate dal punto di vista emozionale e soggettivo. In questo modo, il verso non è più artificio retorico, ma è voce di dentro e musica delle cose (DE SANCTIS, 2002, p. 949).

Anche il lessico utilizzato dai due scrittori rispetta tale criterio altisonante, citando spesso la tradizione letteraria precedente, e creando uno stile alto e ricco di artifici retorici. L'insistenza del fonosimbolismo presente nell'elegia arricchisce il linguaggio con frequenti sinestesie: ad esempio, un "escuro frio" (PESSOA, 2010, p. 48, v. 192) avvolge la notte della nazione portoghese, in attesa del vago chiarore dell'alba e della rinascita. Tale valore fonosimbolico investe anche i versi del Foscolo di una tinta talvolta romantica e orrorosa, come nella descrizione già citata della tomba del Parini, ormai dimenticata tra il buio e l'ululare dell'upupa.

Un ulteriore aspetto formale è l'uso frequente che i due poeti fanno delle antitesi e delle avversative, rivelando in questo modo momenti di disperazione e di sentimenti divisi, superati da entrambi nella fiducia di una sopravvivenza alla fine.

La soluzione che il Foscolo ci offre rispetto alla morte è una risposta laica, in apparente opposizione rispetto alla concezione pessoana: in realtà, le due argomentazioni si conciliano nella fede nell'uomo, ed in particolare, nella figura dell'eroe. Anche la poesia di Pessoa rappresenta infatti una ricerca spirituale di tipo eterodosso rispetto alla teologia cattolica, seguendo quella direttrice esoterica ed iniziatica che accompagna la direzione patriottica nella sua opera. Entrambe le direttrici si esplicano poi a pieno nel suo poema Mensagem, un'epopea del Portogallo a livello storico e mitico. L'epicità e il profetismo del poeta porto- 
ghese lanciano così un ponte tra il finito e l'infinito, tra ciò che è caduco ed effimero e ciò che si rivela eterno. Ed allo stesso modo nel poeta italiano Ugo Foscolo: nonostante la sua posizione rispetto alla morte sia materialistica, questa non può essere inserita in una visione meccanicistica dell'universo, trattandosi piuttosto di una risposta laica all'angoscia della morte che esalta il valore dell'individuo e delle sue azioni sulla terra. Si strappa in questo modo la sacralità della morte alla Chiesa, creando una vera e propria religione dei sepolcri.

In conclusione, se la materia torna alla materia, l'ottimismo della volontà vince il pessimismo della ragione: l'uomo può guadagnarsi un riscatto con le sue qualità, può conquistare una sopravvivenza lasciando un'eredità di affetti, che abbiamo visto nel primo grande trionfo sulla morte della corrispondenza di amorosi sensi. Il lascito del valore civile dell'eroe e dei suoi esempi di alto sentire rappresentano il secondo trionfo sulla morte, nella memoria di una vita terrena valorosa, secondo una visione severamente elitaria. In questa prospettiva il sepolcro, alla cui importanza attentava l'editto di Saint-Cloud, acquistava una funzione sociale come testimonianza di una continuità tra passato e presente, tra defunti e viventi, nella dimensione privata e in quella pubblica e sociale. Se la morte viene sconfitta dalla tomba, l'azione inesorabile del tempo può mettere in pericolo il sepolcro: unico vero risarcimento a tale catena di distruzione e di oblio è la poesia, che può eternare la memoria degli eroi. Levandosi su di un paesaggio di rovine, l'arte vince il silenzio dei secoli, come ultimo grande trionfo sulla morte.

\section{The ode Dei Sepolcri by Ugo Foscolo in a dialogue with the Elegy À memóRia do Presidente-Rei Sidónio Pais by Fernando Pessoa: the victory over death OF POETRY}

Abstract: This work proposes the analysis of the poems Dei Sepolcri, by Ugo Foscolo, and the elegy À memória do Presidente-Rei Sidónio Pais, by the portuguese writer Fernando Pessoa. The authors reflect upon the history of their countries and discover the power of poetry able to defeat death.

Keywords: Ugo Foscolo. Fernando Pessoa. Portuguese literature.

\section{RIFERIMENTI}

BINNI, W. Ugo Foscolo: storia e poesia. Torino: Einaudi, 1982.

DE SANCTIS, F. Storia della letteratura italiana. Milano: Rizzoli, 2002. v. II.

FOSCOLO, U. Dei Sepolcri. In: GROSSER, H.; GUGLIELMINO, S. Il Sistema Letterario 2000: testi del primo Ottocento. Milano: Principato, 2001.

MONTALE, E. Ossi di Seppia. Milano: Mondadori, 2001.

PESSOA, F. Alla Memoria del Presidente-Re Sidónio Pais. Perugia: Edizioni dell’Urogallo, 2010. 